\title{
PERMUTATION POLYNOMIALS IN SEVERAL VARIABLES OVER RESIDUE CLASS RINGS
}

\author{
H. K. KAISER and W. NÖBAUER
}

(Received 21 April 1986)

Communicated by R. Lidl

\begin{abstract}
The concept of a permutation polynomial function over a commutative ring with 1 can be generalized to multiplace functions in two different ways, yielding the notion of a $k$-ary permutation polynomial function $(k>1, k \in \mathbf{N})$ and the notion of a strict $k$-ary permutation polynomial function respectively. It is shown that in the case of a residue class ring $\mathbf{Z}_{m}$ of the integers these two notions coincide if and only if $m$ is squarefree.
\end{abstract}

1980 Mathematics subject classification (Amer. Math. Soc.): 13 B 25.

The representation of permutations by polynomials has been thoroughly studied over the past century. Let $\langle R,+,-, 0, \cdot, 1\rangle$ be a commutative ring with identity. Then we call a function $f: R \rightarrow R$ a permutation permutation function over $\langle R,+,-, 0, \cdot, 1\rangle$ if $f$ is both a permutation of the set $R$ and a polynomial function over $\langle R,+,-, 0, \cdot, 1\rangle$. A polynomial $f(x) \in R[x]$ which induces such an $f$ is called permutation polynomial over $\langle R,+,-, 0, \cdot, 1\rangle$.

A direct generalization of this concept to functions in several variables is not possible, since polynomial functions $f: R^{k} \rightarrow R, k>1$, can never represent a permutation of $R^{k}$, since $R^{k} \neq R$. Hence we have to consider $k$-tuples $\left(f_{1}, \ldots, f_{k}\right)$ of functions $f_{i}: R^{k} \rightarrow R, i=1, \ldots, k$, and we say: a permutation $\pi$ of $R^{k}$ is represented by $\left(f_{1}, f_{2}, \ldots, f_{k}\right)$ if $\pi\left(r_{1}, \ldots, r_{k}\right)=\left(f_{1}\left(r_{1}, \ldots, r_{k}\right), \ldots, f_{k}\left(r_{1}, \ldots, r_{k}\right)\right)$ for all $\left(r_{1}, \ldots, r_{k}\right) \in R^{k}$.

This yields the following generalization of a permutation polynomial function to the case of several variables: $f: R^{k} \rightarrow R$ is called $k$-place permutation polynomial function (in short $P P F$ ) over $R$, if $f$ is a component in a $k$-tuple of $k$-ary

(ㅇ) 1987 Australian Mathematical Society $0263-6115 / 87 \$ A 2.00+0.00$ 
functions over $R$ which represent a permutation of $R^{k}$ and if $f$ is a polynomial function over $\langle R,+,-, 0, \cdot 1\rangle$. Polynomials $f\left(x_{1}, \ldots, x_{k}\right) \in R\left[x_{1}, \ldots, x_{k}\right]$ which induce such a $P P F$ are called permutation polynomials in $k$ variables over $\langle R,+,-, 0, \cdot, 1\rangle$. The set of all $k$-ary $P P F$ over $\langle R,+,-, 0, \cdot, 1\rangle$ is denoted by $S_{k}(R)$. It is easy to see that every $k$-ary $P P F f$ appears as first component in the representation of a suitable permutation of $R^{k}$, hence we have: A polynomial function $f: R^{k} \rightarrow R$ is a $k$-ary $P P F$ over $\langle R,+,-, 0, \cdot, 1\rangle$ if and only if there are $k$-ary functions $f_{2}, \ldots, f_{k}$ over $R$ such that $\left(f, f_{2}, \ldots, f_{k}\right)$ represents a permutation of $R^{k}$.

Another possibility of generalization is the following: A polynomial function $f: R^{k} \rightarrow R$ is called strict permutation polynomial function over $\langle R,+,-, 0, \cdot, 1\rangle$ (in short $S P P F$ ) if there are $k$-ary polynomial functions $f_{2}, \ldots, f_{k}$ over $\langle R,+,-, 0, \cdot, 1\rangle$ such that the $k$-tuple of polynomial functions $\left(f, f_{2}, \ldots, f_{k}\right)$ represents a permutation of $R^{k}$. Again we call a polynomial $f\left(x_{1}, \ldots, x_{k}\right) \in$ $R\left[x_{1}, \ldots, x_{k}\right]$ a strict permutation polynomial over $\langle R,+,-, 0, \cdot, 1\rangle$ if $f\left(x_{1}, x_{2}, \ldots, x_{k}\right)$ induces a $S P P F f$. The set of all $k$-ary $S P P F$ over $\langle R,+,-, 0, \cdot, 1\rangle$ will be denoted by $S S_{k}(R)$. If $P_{k}(R)$ symbolizes the set of all $k$-ary polynomial functions $f: R^{k} \rightarrow R$, then $S S_{k}(R) \subseteq S_{k}(R) \subseteq P_{k}(R)$.

Both generalizations have been investigated in a series of papers (see H. Lausch and W. Nöbauer [1] and bibliography thereto appended). Especially for permutation polynomials over finite fields a number of results are known (see R. Lidl and H. Niederreiter [2]). In the case of finite fields the two notions of $P P F$ and $S P P F$ coincide, since every $k$-ary function over $G F(q)(k \in \mathbb{N}$, arbitrary) with values in $G F(q)$ can be represented by a polynomial function over $G F(q)$. In [3] W. Nöbauer raised the problem for which finite commutative rings this coincidence holds. In this paper we solve the problem for all residue class rings $\mathbb{Z}_{m}$ of the integers.

First we recall some properties of permutation polynomial functions and permutation polynomials over $\langle R,+,-, 0, \cdot, 1\rangle$. Permutation polynomial functions over finite rings can be characterized as follows:

THEOREM. Let $\langle R,+,-, 0, \cdot, 1\rangle$ be a finite commutative ring with identity. A polynomial function $f \in P_{k}(R)$ is a $k$-ary $P P F$ if and only if for every $r \in R$ the set of all solutions in $R$ of the equation $f\left(x_{1}, \ldots, x_{k}\right)=r$ has the cardinality $|R|^{k-1}$.

For a proof see H. Lausch and W. Nöbauer ([1], Chapter 3, Proposition 12.21.).

LEMMA 1. (i) If $f\left(x_{1}, x_{2}, \ldots, x_{k}\right) \in R\left[x_{1}, x_{2}, \ldots, x_{k}\right]$ is a $k$-ary permutation polynomial over $\langle R,+,-, 0, \cdot, 1\rangle$, then $f\left(x_{1}, x_{2}, \ldots, x_{k}\right)$ is an $n$-ary permutation polynomial over $\langle R,+,-, 0, \cdot, 1\rangle$ for every $n>k$. 
(ii) If $f\left(x_{1}, \ldots, x_{k}\right) \in R\left[x_{1}, x_{2}, \ldots, x_{k}\right]$ then we denote by $\bar{f}\left(x_{1}, \ldots, x_{k}\right)$ the polynomial which is obtained by removing the constant term from $f$. Then the following holds: The $k$-tuple $\left(f_{1}\left(x_{1}, \ldots, x_{k}\right), \ldots, f_{k}\left(x_{1}, \ldots, x_{k}\right)\right)$ of $k$-ary polynomials over $\langle R,+,-, 0, \cdot, 1\rangle$ induces a permutation of $R^{k}$ if and only if $\left(f_{1}\left(x_{1}, \ldots, x_{k}\right)\right.$, $\left.\bar{f}_{2}\left(x_{1}, \ldots, x_{k}\right), \ldots, \bar{f}_{k}\left(x_{1}, \ldots, x_{k}\right)\right)$ does so as well.

Proof. (i) follows easily from the preceding theorem and (ii) is evident.

Now we turn to the problem of finding all $\mathbb{Z}_{m}$ for which every $f \in \mathbb{Z}_{m}$ is a $P P F$ if and only if $f$ is a $S P P F$. Let $m=p_{1}^{e_{1}} \cdot p_{2}^{e_{2}} \cdot \ldots \cdot p_{n}^{e_{n}}$ be the prime factorization of $m$. Then $\mathbf{Z}_{m}$ is isomorphic to the product of the residue class rings $\mathbf{Z}_{p_{i}^{e_{i}}}, i=1, \ldots, n$. The following theorem is taken from $\mathbf{H}$. Lausch and $\mathrm{W}$. Nöbauer [1] (Chapter 3, Proposition 12.43).

THEOREM. If $\mathbf{V}$ is the variety of commutative rings with identity and $R=A \times B$ in $\mathbf{V}$ then there is a bijective mapping of $S S_{k}(R)$ onto $S S_{k}(A) \times S S_{k}(B)$ which is, if $R$ is finite, the restriction of a bijective mapping from $S_{k}(R)$ onto $S_{k}(A) \times S_{k}(B)$.

This theorem reduces our study to $\mathbf{Z}_{p^{e}}, p$ prime, $e \in \mathbb{N}, e>1$ (since for finite fields $\mathbf{Z}_{p}$-as mentioned above-every $P P F$ is $S P P F$ ). First we consider the case $k=2$ :

We show that in this case there are $P P F$ over $\mathbb{Z}_{p^{e}}$ which are not $S P P F . \varphi$ denotes Euler's phi-function.

LEMMA 2. The binary function $f: \mathbf{Z}_{p^{e}}^{2} \rightarrow \mathbb{Z}_{p^{e}}, p$ prime, $e>1$, defined by $f(x, y)$ $=p x+y^{\Phi\left(p^{e}\right)+1} \bmod p^{e}$ is a PPF.

Proof. If we multiply every $x \in \mathbb{Z}_{p^{e}}$ by $p$, we obtain $\left(\bmod p^{e}\right)$ every non negative multiple of $p$ which is smaller than $p^{e}$ exactly $p$ times. If $\left(y, p^{e}\right)=1$ then

$$
y^{\varphi\left(p^{e}\right)+1}=y^{p^{e}-p^{e-1}+1} \equiv y \quad \bmod p^{e}
$$

by the theorem of Fermat-Euler. If $\left(y, p^{e}\right)>1$ then

$$
y^{\varphi\left(p^{e}\right)+1}=y^{p^{e}-p^{e-1}+1} \equiv 0 \quad \bmod p^{e}
$$

since we have $p^{e}-p^{e-1}+1>p^{e}-p^{e-1} \geqslant 2 p^{e-1}-p^{e-1}=p^{e-1} \geqslant$ $(1+1)^{e-1}=\sum_{i=0}^{e-1}\left({ }^{e-1} i\right) \geqslant e$. Let $a \in \mathbf{Z}_{p^{e}}$ be a fixed element and $a \equiv t \bmod p$, $t \in\{0, \ldots, p-1\}$. Then we obtain by $f(x, a)$ every element of $\mathbf{Z}_{p^{e}}$ which is congruent $t \bmod p$ exactly $p$ times, if $x$ runs through the whole of $\mathbf{Z}_{p^{e}}$. Since the 
congruence $y^{\varphi\left(p^{e}\right)+1} \equiv i \bmod p(i=0,1, \ldots, p-1)$ has $p^{e-1}$ incongruent solutions $\bmod p^{e}$, we have: The equation $f(x, y)=u$ possesses $p \cdot p^{e-1}=p^{e}$ solutions in $\mathbb{Z}_{p^{e}}$ for every $u \in \mathbb{Z}_{p^{e}}$. Hence $f$ is a binary $P P F$ over $\mathbb{Z}_{p^{e}}$.

Lemma 3. $f: \mathbb{Z}_{p^{e}}^{2} \rightarrow \mathbb{Z}_{p^{e}}$, defined by $f(x, y)=p x+y^{\varphi\left(p^{e}\right)+1}$ for all $(x, y) \in \mathbf{Z}_{p^{e}}^{2}$ is not SPPF.

Proof. We denote $f^{-1}(0) \subseteq \mathbf{Z}_{p^{e}}^{2}$ by ${ }_{f} N_{0}$. For the function $f$ defined in Lemma 2 we have

$$
{ }_{f} N_{0}=\left\{0, p^{e-1}, 2 p^{e-1}, \ldots,(p-1) p^{e-1}\right\} \times\left\{0, p, 2 p, \ldots,\left(p^{e-1}-1\right) p\right\} .
$$

Since $\left|{ }_{f} N_{0}\right|=p^{e}$, a necessary condition for $f$ to be a binary $S P P F$ is the existence of a polynomial function $g: \mathbf{Z}_{p^{e}}^{2} \rightarrow \mathbf{Z}_{p^{e}}$ such that $g$ restricted to $N_{0}$ is a mapping onto $\mathbb{Z}_{p^{\text {e }}}$. But such a $g$ does not exist, since for any polynomial $g(x, y)$ with constant term $c$, we have $g(\xi, \eta) \equiv c \bmod p$ for all $(\xi, \eta) \in_{f} N_{0}$.

To settle the general case let $k>2$ be a fixed integer. Then $f: \mathbb{Z}_{p^{e}}^{k} \rightarrow \mathbb{Z}_{p^{e}}$, defined by $f\left(x_{1}, \ldots, x_{k}\right):=p x_{1}+x_{2}^{\varphi\left(p^{e}\right)+1}$ for all $\left(x_{1}, \ldots, x_{k}\right) \in \mathbb{Z}_{p^{e}}^{p^{e}}$, is by Lemma 2 and Lemma 1 , (i) a $k$-ary $P P F$ over $\mathbb{Z}_{p^{e}}$ and ${ }_{f} N_{0}=$ $\left\{0, p^{e-1}, 2 p^{e-1}, \ldots,(p-1) p^{e-1}\right\} \times\left\{0, p, 2 p, \ldots,\left(p^{e-1}-1\right) p\right\} \times \mathbb{Z}_{p^{e}}^{k-2}$.

\section{LemMa 4. The function $f$ defined above is not a SPPF over $\mathbb{Z}_{p^{e}}$.}

Proof. Let us assume in the contrary that $f$ is $S P P F$. Then there are $\varphi_{2}, \ldots, \varphi_{k} \in P_{k}\left(\mathbb{Z}_{p^{e}}\right)$ such that $\left(f, \varphi_{2}, \ldots, \varphi_{k}\right)$ represents a permutation of $\mathbb{Z}_{p^{e}}^{k}$ and all $\varphi_{i}, i=2, \ldots, k$ are assumed to be without constant term. If we restrict $f$, $\varphi_{2}, \ldots, \varphi_{k}$ to ${ }_{f} N_{0}$ then $\left(f, \varphi_{2}, \ldots, \varphi_{k}\right)$ has to represent all $k$-tuples over $\mathbb{Z}_{p^{e}}$ with first component 0 . Thus $\left(\varphi_{2}, \ldots, \varphi_{k}\right)$ has to represent each element of $\mathbb{Z}_{p^{e}}^{k-1}$ if $\left(x_{1}, x_{2}, \ldots, x_{k}\right)$ runs over ${ }_{f} N_{0}$. Hence $\left(\varphi_{2}, \ldots, \varphi_{k}\right)$, if considered mod $p$, represents all the elements of $\mathbb{Z}_{p}^{k-1}$, if $\left(x_{1}, x_{2}, \ldots, x_{k}\right)$ runs over $N_{0}$.

Each polynomial $\varphi_{i}$ can be written as $g_{i}\left(x_{3}, x_{4}, \ldots, x_{k}\right)+h_{i}\left(x_{1}, x_{2}, \ldots, x_{k}\right)$, where every term of $h_{i}\left(x_{1}, x_{2}, \ldots, x_{k}\right)$ has a factor $x_{1}$ or $x_{2}$. Since $x_{1} \equiv x_{2} \equiv$ $0 \bmod p$ for all $\left(x_{1}, x_{2}, \ldots, x_{k}\right) \in_{f} N_{0}$, we obtain $\varphi_{i}\left(x_{1}, x_{2}, \ldots, x_{k}\right) \equiv$ $g_{i}\left(x_{3}, x_{4}, \ldots, x_{k}\right) \bmod p$ for every $\left(x_{1}, x_{2}, \ldots, x_{k}\right) \in_{f} N_{0}$. Hence $\left(\varphi_{2}, \ldots, \varphi_{k}\right)$ has at most $\left|\mathbb{Z}_{p^{\mathrm{e}}}\right|^{k-2}$ distinct values $\bmod p$ if $\left(x_{1}, x_{2}, \ldots, x_{k}\right)$ runs over ${ }_{f} N_{0}$, a contradiction. Hence $f$ is not a $k$-ary $S P P F$ over $\mathbb{Z}_{p^{e}}$.

This yields the following

THEOREM. Let $R$ be a finite commutative ring with identity which is isomorphic to a direct product $\mathbb{Z}_{p_{1}^{e_{1}^{1}}} \times \mathbb{Z}_{p_{2}^{e^{2}}} \times \cdots \times \mathbb{Z}_{p_{n}^{e^{n}}}\left(p_{i}, i=1, \ldots, n\right.$, not necessarily distinct primes, $e_{i} \in \mathbb{Z}, e_{i} \geqslant 1$, for $\left.i=1, \ldots, n\right)$. Then every $k$-ary $P P F$ is $\operatorname{SPPF}(k>1$, $k \in \mathbb{N}$ arbitrary) if and only if all $e_{i}=1$. 
COROllaRY. Let $\mathbf{Z}_{m}$ be a residue class ring of the integers. Then every $k$-ary $P P F$ is $S P P F$ if and only if $m$ is squarefree.

\section{References}

[1] H. Lausch and W. Nöbauer, Algebra of polynomials (North-Holland, Amsterdam, 1973).

[2] R Lidl and H. Niederreiter, Finite fields (Addison-Wesley, Reading, Massachusetts, 1983).

[3] W. Nöbauer, 'Darstellung von Permutationen durch Polynome und rationale Funktionen', Berichte des Math. Forschungsinst. Oberwolfach 5 (1971), 89-100.

Institut für Algebra und Diskrete Mathematik

Technische Universität Wien

Wiedner Hauptstraße 8-10

A-1040 Vienna, Austria 